\title{
(Re)Focussing on behavioural change: An examination of the utility of Hidden Markov Modelling
}

Journal of Social Marketing [in press]

\author{
Patricia David \\ Social Marketing @ Griffith, Griffith University \\ Sharyn Rundle-Thiele \\ Social Marketing@ Griffith, Griffith University \\ Jason Pallant \\ Department of Management and Marketing, Swinburne University of Technology
}

\begin{abstract}
Purpose - Behavioural change practice has focussed attention on understanding behaviour; failing to apply dynamic approaches that capture the underlying determinants of behavioural change. Following recommendations to direct analytical focus towards understanding both the causal factors of behaviour and behavioural change to enhance intervention practice, this paper applied a Hidden Markov Model (HMM) approach to understand why people transition from one state to another (e.g. reporting changes from wasting food to not wasting food or vice versa).

Design/methodology/approach - Data was drawn from a 2017 food waste program ${ }^{1}$ that aimed to reduce waste of fruit and vegetables by increasing self-efficacy through a 2-week pilot, featuring recipes and in-store cooking demonstrations. A repeated measure longitudinal research design was used. 314 households completed a phone survey prior to the two-week pilot and 244 completed the survey in the weeks following the intervention (77\% retention in the evaluation study).

Findings - Two behavioural states were identified, namely fruit and vegetable (FV) wasters and non FV-wasters. Age was identified as a causal factor for FV food wasting prior to the campaign (45-54 years were most likely to waste FV). Following the intervention, a total of $43.8 \%$ transitioned away from FV wasters to non-wasters, and attitudes and self-efficacy were indicated as potential causal factors of this change in FV waste behaviour.

Originality/value - Through this application, it is demonstrated how HMM can identify behavioural states, rates of behaviour change and importantly how HMM can identify both causal determinants of behaviour and behavioural change. Implications, limitations and future research directions are outlined.
\end{abstract}

Keywords Behaviour, behaviour change, social marketing, Hidden Markov Modelling, food waste Paper type Research paper

\footnotetext{
${ }^{1}$ Waste Not Want Not was delivered in Redland City Council by Social Marketing @ Griffith staff and students, namely Dr. Kathy Knox, Ms. Jeawon Kim and Mr. Sam Hodgkins. The authors acknowledge this team as data set owners.
} 


\section{Background}

As noted by Kriznik et al. (2018) "the dominant epistemic assumption (i.e. the assumption about what constitutes admissible evidence to guide action) underpinning the implementation of public health and other behavioural change programmes is that human behaviour is a major determinant of health, and that behaviour is largely a matter of individual choice; individuals are therefore responsible for their own health and for making health-related behaviour changes". Evolving from commercial marketing, social marketing first emerged as a discipline within health which aimed at changing individual behaviours to benefit both the individual and the society in which they live (Andreasen, 2002). In line with other behavioural change and public health practice recent social marketing reviews highlight the continued dominant focus of intervention efforts on the individual (for example see Almestahiri et al., 2017), a focus that may overlook social and environmental behavioural determinants. It is acknowledged that an individual focus is frequently stigmatising and can lead to victim-blaming (Brennan et al., 2016).

In an individually (also referred to as downstream or micro) focussed program, social marketing uses the tools and techniques of commercial marketing to influence the individual whose behaviour needs to change to benefit society more broadly. Andreasen explains that what sets social marketing apart from other behaviour change fields are three factors: first, behaviour (and not awareness raising) is at the core; second, social marketing is customerdriven; and perhaps most notably, social marketing creates attractive programs into which individuals will willingly enter into exchange to achieve the desired behaviour (Andreasen, 2002). Social marketing's core principle of exchange overcomes victim blaming and stigmatisation criticisms directed at behavioural change programs, particularly when monetary exchange occurs and people willingly purchase a program of their own volition.

Typically in social marketing, behavioural change is the desired ultimate goal. There are some settings where behaviour maintenance, and not behavioural change are required (for example see Rundle-Thiele et al., 2015 who sought to maintain the non-alcohol drinkers while attempting to moderate binge drinkers). Measurement indicating that behavioural change has occurred following implementation would indicate to program evaluators that the social marketing program was a success. This suggests that in many cases behaviour change is the variable of interest for social marketers, and not simply behaviour. 
Although behaviour change is the end goal of social marketing dominant use of cross-sectional designs is noted in a review of the literature (for example, Huchting et al., 2008, Armitage, 2005), which focuses understanding on behaviour and not behavioural change. Use of longitudinal approaches are less evident (for example, Leatherdale et al., 2014, Mihrshahi et al., 2015, Shefferly et al., 2016) and therefore focus on behavioural change remains limited within social marketing. Further, theoretical perspectives are largely behaviour and not behavioural change focussed (Brennan et al., 2014). Consistent with prevailing theoretical views; when longitudinal approaches are used, research focus remains centred on identifying the causal determinants of behaviour rather than examining the determinants of behavioural change. Researchers contends that interventions are more effective when they are aimed at causal determinants of both behaviour and behaviour change (Davis et al., 2015, Michie et al., 2008). As behaviour change is the end goal of social marketing, there is a need for greater understanding of the causal determinants of behaviour change.

In addition to a lack of focus on behavioural change, prevailing practice relies on analysis methods that are static in nature. For example, Repeated Measure ANOVAs are frequently employed in outcome evaluations in social marketing (for an example see Schuster et al., 2016). A shift towards dynamic evaluation methods is needed to centre research attention on the proportion of the target audience who change (or not) and understanding which factors support positive change outcomes along with the factors contributing to undesired change. Responding to calls to direct efforts to understanding more complex relational and dynamic factors (Kriznik et al., 2018), this paper employs one dynamic analytical method drawing on available data from a social marketing food waste pilot program. Food waste is a global problem. It is estimated that one third of all food supplied for human consumption is wasted (Stancu et al., 2016), hence, there is an urgent need for effective interventions focussed on changing food wasting behaviour to be put in place. The aims of this paper are twofold. First, this paper seeks to explore the utility of employing Hidden Markov Modelling to understand transition states, which involves understanding why people transition from one state to another (e.g. wasting food to not wasting food or vice versa). Second, consistent with dominant research practices this study aims to extend our understanding of the causal determinants of food waste behaviour.

\section{Literature Review}

The most commonly used behavioural theories reported in social marketing research are Social Cognitive Theory (Bandura, 1986), Theory of Planned Behaviour (Ajzen, 1991), Health Belief 
Model (Rosenstock, 1974) and Stages of Change Model (Prochaska et al., 1994) (see Truong and Dang, 2017). Close inspection of the theories applied within social marketing indicates the main focus of these commonly adopted theories is the explanation and description of behaviour at one point in time or an individual's readiness for change as measured at a point in time (static). For example, commonly used theories such as the Theory of Planned Behaviour aim to explain or predict behaviour at one single point in time. For example, the author of the Theory of Planned Behaviour (TPB) acknowledges that "the TPB is in fact not a theory of behaviour change" (Ajzen, 2015). Instead, TPB was developed to help researchers explain and predict people's (current) intentions and behaviour (Ajzen, 2015).

One model known for its focus on behavioural change is the Stages of Change Model (also referred to as the Transtheoretical Model) (Prochaska et al., 1994). Stages of Change focuses research attention on identifying different states. Specifically, Stages of Change centres on identifying a person's readiness for change. Although the model is change focussed, its application frequently captures readiness at a single point in time providing an understanding of the proportion of individuals at each stage of change. However, the model does not clearly identify which factors may cause a person to transition (change) from one readiness state to another in a single diagnostic model. Advances in methodologies using longitudinal data permit transitions between states to be modelled. Crucially, dynamic models can explain and predict how individuals change (or not), while also identifying the determinants of positive change, negative change, or lack of change. Given behaviour change is the end goal of social marketing, behavioural change (a dynamic process) and not behaviour (a static phenomenon) should be the analytical focus. For behavioural change to be assessed, variables have to be consistently measured at different time points, in exactly the same way, to ensure reliability (Kher and Serva, 2014) and evaluation methodologies need to be dynamic, capturing changes over time (Ployhart and Vandenberg, 2010), which occurs in repeated measure longitudinal research designs.

Commonly used behavioural theories such as the Theory of Planned Behaviour aggregate (average) past behaviour overlooking individual variation. Yet individual behaviour change is a dynamic process, where ongoing changes both internally and externally can lead to growth, regression, or stagnation. Research focused on explaining or predicting phenomena at a single point in time is not able to capture these dynamic effects. An example is the study undertaken by Gardner et al. (2012), where despite having measured all items across two time points (T1 and T2) use of change data (calculation of difference scores) was overlooked, ignoring the 
potential for a dynamic approach to be taken. Gardner et al. (2012) used behaviour at time point 2 as the dependent variable and independent variables at time point 1 (past behaviour, intention, attitudes, subjective norms, PBC, self-identity and habit). This approach is typical of the predominant research practice in behavioural change science, and it fails to recognise that behaviour change is a dynamic process (Ployhart and Vandenberg, 2010, Jebb et al., 2015, Wang et al., 2017). As noted above, interventions are more effective when they are aimed at causal determinants of not only behaviour but also behaviour change. Achieving this aim requires consideration of behaviour change as a dynamic, and somewhat individual, process. Taken together, a review of the social marketing literature indicates the need for theoretical and methodological development to examine behaviour change as a dynamic process. Against this background, this paper examines the utility of the Hidden Markov Model as one dynamic method that can potentially be employed to evaluate the effectiveness of a two-week pilot food waste program.

\section{Method}

\section{Design}

A convenience sample of 314 local council area residents were allocated into either the program $(n=110)$ or control groups $(n=204)$. The Waste Not Want Not $^{2}$ program group received an intervention pack which consisted of a shopping bag, chopping board, 16 new leftover reuse recipe cards, invitation flyer, and a shopping list. Further, residents had access to a 2-week interactive shopping centre display between the pre and post-survey period. This display was delivered to engage the local community to trial developed recipes. The interactive shopping centre display consisted of daily food demonstrations delivered by a chef, interaction with volunteers and a display fridge, culminating in a Cook-Off event delivered by two local Chefs.

A controlled, repeated measure design was used to assess social marketing pilot program outcomes. A telephone survey was conducted in March (before the campaign) and April 2017 (two weeks after the campaign) following ethical and local government approval. The survey was conducted using Computer-Assisted Telephone Interviewing (CATI) equipment. Personal information including postcode and address were essential for distribution of campaign materials and were used to send the household intervention package to the program group. Self-

\footnotetext{
${ }^{2}$ Waste Not Want Not was a food waste pilot program that was implemented in the Redland City Council community in March 2017. The program aimed to increase use of left over foods.
} 
efficacy in reusing leftovers (Waste and Resources Action Programme, 2007, Waste and Resources Action Programme, 2010), attitudes (Fishbein and Ajzen, 2011), social norms (Perugini and Conner, 2000, Rhodes and Courneya, 2003), and self-reported fruit and vegetable waste were measured pre and post program. Self-efficacy items captured a range of behaviours linked to food waste including planning meals, storing food correctly, planning food shopping, buying the right amount of food, measuring portion size, transforming leftovers, reusing leftovers and storing leftovers correctly. The proportion of fruit and vegetable waste was measured in the pre and post surveys using the same categorical measure, where 1 was hardly any, 2 was less than 10 per cent, 3 was between 11 and 25\%, 4 was between $26 \%$ and $50 \%$ and 5 was more than $51 \%$. Respondents were asked to provide an approximation of how much fruit and vegetable waste was thrown away in their household of the food bought, cooked and/or grown, in the past week. Demographic information including age, gender and private garden was collected at baseline only. Reported exposure to the intervention was also recorded, to ensure that the program group had successfully received the intervention materials.

\section{Participants}

Some attrition (33 per cent) was observed for the post-campaign survey. Thus, there were 244 (77 per cent retention) participants that completed both pre and post surveys. Of those, 91 were from the program group (82.7 per cent retention) who received a household intervention pack and/or saw the shopping centre display and 153 were from the control group who did not receive the intervention pack and who were not exposed to the local shopping centre display (75 per cent retention). Retention rates were high relative to typical social research. One issue with longitudinal studies is the loss of participants over time. In order to avoid high attrition rates, incentives to participate in the surveys (pre and post) were offered. For the control group, a chance to participate in a draw to win a $\$ 50$ gift voucher was offered, and the intervention group received a free program pack valued at $\$ 100$. Respondents were asked a series of questions to provide a profile of the sample obtained in the survey. Over $73 \%$ of the sample were female. The majority of the participants belonged to the 34 to 49 year old age group, and described their households as a family (only adults) and having a private garden.

\section{Data Analysis}

A Hidden Markov Model (HMM), was used to examine the effectiveness of the food waste campaign. Hidden Markov Models, which are often referred to as Latent Transition Analysis 
in health research, can be summarised as a form of longitudinal segmentation (Collins and Lanza, 2010). That is, the HMM categorises individuals into groups at different points in time. Importantly, these groups are dynamic meaning that any given individual may be a member of a certain group at one point in time, but then may belong to a different group at a different point in time (Collins and Lanza, 2010). Importantly, this means the HMM not only reveals groups (i.e. segments) within a population, but also examines the way individuals move between these groups.

HMMs have been successfully applied in a broad range of settings involving individual behaviour. Netzer et al. (2008) apply a HMM to alumni-university interactions, finding three behaviour groups ranging from Dormant, to Occasional, to Active donors. Importantly, they demonstrated that individuals may move between these groups, and that this is affected by factors such as attendance at reunion events, and volunteering. Singh et al. (2011) utilised HMM to investigate learning dynamics in the context of open source software projects. Their model revealed three learning groups (low, medium, and high) which represented different learning patterns. They also find that learners may move between these groups based on the learning activities they engage with. Finally, in a health-setting, Chung et al. (2005) applied HMM (labelled as Latent Transition Analysis) to investigate the dynamics of substance use behaviour among adolescent females. They found five groups based on substance use, ranging from no use, to alcohol or cigarette use in isolation, to a combination of substances. Their model shows that the likelihood of progressing to more advanced substance use depends on stage of puberty, and the age when substance use began. These examples serve to highlight the utility of HMM in uncovering 1) behavioural groups, 2) the rates of change between groups, and most importantly 3) the determinants of change. Detailed discussion including practical applications and examples of HMM are available in Visser (2011) and Collins and Lanza (2010).

The HMM makes a number of assumptions to examine the way individuals evolve over time in a dynamic way. First, consistent with Factor Analysis and Structural Equation Modelling, variation among observed variables is assumed to be caused by differences within a latent (unobserved) variable (Collins and Lanza, 2010). However, HMM is distinct from these methods as the second assumption is that this latent variable is assumed to be categorical, rather than continuous (Collins and Lanza, 2010). Hence, a HMM proposes that there are sub-groups (or segments) of individuals which differ in ways that cause differences to occur in observed variables. To illustrate this, consider the example of Chung et al. (2005) discussed above. In 
their case, the HMM assumes that there are sub-groups of adolescent females regarding substance abuse behaviours. However, these groups are not known or observed. Instead, membership in these groups is inferred based on observed behaviour. That is, through the data individuals are observed to use cigarettes at a given point in time. From this observed behaviour, it can then be inferred that this particular individual is in a state of "substance user" at that point in time.

This example also helps lead to the final assumption of the HMM, which is that the latent groups are dynamic. This means individuals may change groups over time (Langeheine and Van de Pol, 2002). Following the substance use example, an individual who is observed to be a "substance user" may be found at a later date to no longer use cigarettes. That is, they have changed from a group of substance use, to a group of non-substance use. To signify the dynamic nature, the sub-groups in a HMM are referred to as "latent states", and "transitions" refer to the movement from one state to another over time (Langeheine and Van de Pol, 2002, Vermunt, 2004). These assumptions make HMM an appropriate method for our study. Given our context, the HMM assumes that there are dynamic subgroups of individuals in relation to food waste behaviour, and investigates movement between these groups (i.e. behaviour change).

As discussed above, observed variables, are used to describe the latent states in a HMM. These are known as "indicator variables" (Collins and Lanza, 2010). Changes in individual scores on these variables are also used to infer transitions between latent states. In our study, a single indicator variable Proportion of Fruit and Vegetable (FV) Wasted, which measured the proportion of fruit and vegetables that a family wasted in the past week as a categorical variable. This variable had three categories; hardly any, less than $10 \%$, and $10 \%$ or more. Repeated measurement occurred pre and post intervention. Hence the proportion of FV Waste at two time points was included; prior to the intervention, and after the intervention. This variable was measured at an individual level, allowing us to track changes (transitions) over time.

HMM can also incorporate variables to test the causal factors of the latent states individuals belong to, and the way they transition between states. These variables are referred to as “covariates" (Chung et al., 2005, Collins and Lanza, 2010). The impact of covariates is tested in a similar way to logistic regression, where the covariate may have a positive or negative impact on the likelihood of an individual being in a given latent state, and/or transitioning between states (Chung et al., 2005, Collins and Lanza, 2010, Vermunt, 2004). In this study, an 
examination of evolving FV food waste behaviour is undertaken while testing the effectiveness of a social marketing campaign. Hence, our covariates include exposure to the campaign as well as other variables which may impact changes to food waste behaviour. A full list of variables included in our model is displayed in Table 1.

\section{Table 1. Variables included in HMM}

\begin{tabular}{|c|c|}
\hline Variable & Description \\
\hline \multicolumn{2}{|l|}{ Indicators } \\
\hline Proportion of & The proportion of food a family wasted in the past week; Hardly any, \\
\hline FV wasted & less than $10 \%$, or $10 \%$ or more \\
\hline \multicolumn{2}{|l|}{ Covariates } \\
\hline Exposure & Binary indicator of whether an individual was exposed to the campaign \\
\hline Age & The age of the respondent (categorical) \\
\hline Gender & The gender of the respondent (dichotomous) \\
\hline PrivateGarde & Binary indicator of whether the respondent had access to a private \\
\hline $\mathrm{n}$ & garden \\
\hline Attitude & $\begin{array}{l}\text { Rating of attitudes towards food waste, measured pre and post } \\
\text { campaign }\end{array}$ \\
\hline SelfEfficacy & $\begin{array}{l}\text { Rating of self-efficacy towards food waste, measured pre and post } \\
\text { campaign }\end{array}$ \\
\hline SocialNorm & $\begin{array}{l}\text { Rating of social norms towards food waste, measured pre and post } \\
\text { campaign }\end{array}$ \\
\hline
\end{tabular}

\section{Results}

Descriptive Analysis

Descriptive statistics for key variables pre and post campaign were analysed across our entire sample, and are displayed in Table 2. 


\begin{tabular}{cll}
\hline & $\begin{array}{l}\text { Pre } \\
\text { Campaign } \\
\mathbf{N = 2 4 4}\end{array}$ & $\begin{array}{l}\text { Post } \\
\text { Campaign } \\
\mathbf{N = 2 4 4}\end{array}$ \\
\hline Proportion of FV wasted & & \\
$\quad$ Hardly any & $45.1 \%$ & $55.7 \%$ \\
Less than 10\% & $38.5 \%$ & $31.1 \%$ \\
10\% or more & $16.4 \%$ & $13.1 \%$ \\
Attitudes & 2.3 & 2.2 \\
Self-Efficacy & 5.7 & 5.7 \\
Social Norms & 5.6 & 5.6 \\
\hline
\end{tabular}

Across our sample, it can be observed that the proportion of individuals who reported wasting "hardly any" food or vegetables prior to the campaign (45.1\%) increased after the campaign (55.7\%). Additionally, the proportion who wasted $10 \%$ or more decreased from $16.4 \%$ to $13.1 \%$. Hence, at an overall level positive changes in the amount of fruit and vegetables wasted after the campaign were observed, which indicates "model-free" evidence of changes to behaviour over time. Notably, there is a $10 \%$ increase in the proportion of households reporting they waste hardly any food after the campaign. Interestingly, the average scores on the Attitudes, Self-Efficacy, and Social Norms scales are relatively consistent prior to and post the campaign.

\section{Hidden Markov Model}

The "Hidden" aspect of the HMM refers to the fact that the underlying states of behaviour in the model are not known prior to analysis (Visser, 2011). Importantly, this extends not only to the profile or nature of the states, but also the number of states. That is, it is not known how many states there are, or what each state entails. To rectify this, models with varying numbers of states, as well as different state profiles, are estimated. A likelihood function is then used to determine the number and profile of states that best fits the observed data (Visser, 2011). In other words, the preferred solution is the one that creates the highest likelihood that the observed data would have occurred (Collins and Lanza, 2010).

To generate our solution, Hidden Markov Models were estimated with ranging numbers of states using LatentGold Version 5 (Vermunt and Magidson, 2013). Full details regarding how 
LatentGold estimates a Hidden Markov Model can be found in the technical guide provided by Vermunt and Magidson (2013). For each possible number of states (i.e. models with 1-state, models with 2-states etc.) the model was started with 100 different random sets of parameters (i.e. different cluster profiles). Following best practice, a combination of ExpectationMaximisation and Newton-Raphson algorithms was used to estimate the best possible parameters (state profiles) based on each set of starting values (McCutcheon, 2002). Estimating the model based on multiple sets of starting parameters provides a way of ensuring the model converges on the true best (or "global") solution, rather than a sub-optimal (or "local) solution based on the starting point used.

We then compare the solutions ranging from 1-5 states using The Bayesian Information Criterion (BIC). The BIC compares the fit of the generated solution with the observed data, while also imposing a penalty for more complex models (Masyn, 2013). The model with the lowest BIC value is generally preferred, as lower numbers represent a better model fit while accounting for model complexity (Masyn, 2013). Table 3 displays the BIC statistic for models ranging from 1 state to 5 states, as well as the Log-Likelihood (LL) value on which the BIC is based.

Table 3. Model fit statistics

\begin{tabular}{lll}
\hline Model & LL & BIC \\
\hline 1-state & -485.6 & 1015.1 \\
2-states & -419.7 & 998.7 \\
3-states & -379.8 & 1144.3 \\
4-states & -345.5 & 1411.1 \\
5-states & -325.8 & 1816.9 \\
\hline
\end{tabular}

The data indicated that the BIC was lowest with two states, which suggests that two states of behaviour provided the best combination of model fit and model complexity. Hence, this is the first important outcome of our HMM; there are two states of behaviour related to food waste. The next crucial step is to consider what these two behavioural states are. The answer to this comes by examining the profile of each state on the indicator variables included in the model (Collins and Lanza, 2010). Table 4 displays the profile of the two revealed states on our indicator variable (Proportion of FV wasted). As our indicator variable is categorical, the profile displays the proportion of members of each state that are expected to be in each 
category. The state profiles are estimated through a density function of responses to the included indicator variables, conditional on membership in the various latent states (Vermunt and Magidson, 2013). A separate probability is estimated for state membership, which can be used to identify the relative size of each state (Vermunt and Magidson, 2013).

Table 4. State profiles

\begin{tabular}{lll}
\hline & State 1 - Non-Wasters & State 2 - Wasters \\
\hline Size & $68.5 \%$ & $31.5 \%$ \\
Proportion of FV wasted & & \\
Hardly any & 0.73 & 0.04 \\
Less than $10 \%$ & 0.26 & 0.52 \\
$11 \%+$ & 0.01 & 0.44 \\
\hline
\end{tabular}

State 1 equates to $68.5 \%$ of our sample and almost two-thirds of this state waste "hardly any" food, while the remainder waste "less than $10 \%$ ". Hence, state 1 refers to respondents with low self-reported fruit and vegetable wastage behaviour. As a result, this state was labelled "nonwasters". State 2 is a smaller state, at only $31.5 \%$ and in contrast to state 1 , only a small proportion (4\%) of state 2 waste "hardly any food". Instead, almost half (44\%) respondents in this group report wasting more than $10 \%$ of their food. Hence, this state was labelled "wasters". Therefore, a key outcome of our HMM is the identification of two states of behaviour (wasters and non-wasters), as well as the approximate sizes of these two groups.

The next outcome of a HMM is the transition probabilities; which estimate the probability of individuals moving between states, compared to the probability of individuals not changing their behaviour (Collins and Lanza, 2010). In other words, the rates of change between the behaviour states are revealed by the model, and are therefore a key result. Transition probabilities are generated by estimating the probability of state membership at a given time period $(t)$ as dependent on state membership in the previous time period $(t-1)$ (Vermunt and Magidson, 2013). This is the "Markov" component of a Hidden Markov Model (Visser, 2011). Recall that our HMM revealed two states of behaviour; waster, and non-waster. Also recall that our model includes two time periods; prior to the intervention, and after the intervention. As a result, the transition probabilities in our model are relatively simple. They represent the probability of individuals who were wasters prior to the campaign becoming non-wasters (relative to no change in behaviour), and vice versa. The transition probabilities estimated by the model are displayed next. 
Table 5. Transition probabilities

\begin{tabular}{llll}
\hline & & \multicolumn{2}{c}{ State after campaign (t) } \\
\hline \multirow{2}{*}{$\begin{array}{lll}\text { State before } \\
\text { campaign (t-1) }\end{array}$} & Non-waster & Waster \\
& Waster & 0.438 & 0.053 \\
\hline
\end{tabular}

In Table 5, the rows represent the possible states of behaviour prior to the campaign. The first row represents individuals who were non-wasters prior to the intervention campaign, while the second row includes wasters. The columns represent the possible states of behaviour after the intervention campaign. The cells on the diagonals therefore represent the likelihood of individuals remaining in the same state over time, while the non-diagonals represent the probability of transitions between states (behaviour change).

To interpret the transition probabilities, it is easiest to read across the rows, as they sum to $100 \%$. By considering the first row, findings show that the probability of individuals who were non-wasters prior to the intervention remaining non-wasters after the campaign (i.e. staying in the same state) is $94.7 \%$ (see Figure 1). There is only a $5.3 \%$ probability that these individuals will transition to the wasters state after the campaign. That is, it is highly likely that non-wasters will remain non-wasters. In contrast, by considering the second row (and the second column) the probability of wasters remaining wasters is substantially lower (56.2\%). In contrast, the probability of this state transitioning (changing behaviour from waster to non-waster) is relatively high $(43.8 \%)$. Hence, these transition probabilities highlight an overall trend of positive behavioural change post intervention.

Next, the factors that influenced individual transition states were examined by considering model covariates. Covariates may have two different impacts in a HMM; influencing an individual's starting state (behaviour prior to the intervention), or influencing transitions between states (change in behaviour over time). In each case, they are tested through a form of logit model as part of the Hidden Markov Model (Collins and Lanza, 2010). Table 6 depicts results of the covariates tested in relation to each individual's starting state. As our model includes only two states, these results display the probability of belonging to State 2 (waster), as opposed to belonging to State 1 (non-waster) as a result of each covariate. 
Table 6. Impact of covariates on starting states

\section{Covariate}

\section{Impact on probability of starting state $=$}

\section{waster}

Displayed as Coefficient (Standard Error), ${ }^{\wedge} \mathrm{p} \leq 0.1,{ }^{*} \mathrm{p} \leq 0.05,{ }^{* *} \mathrm{p} \leq 0.01,{ }^{* * *} \mathrm{p} \leq 0.001$

Results indicate that individuals aged 45-54 are relatively more likely than other age groups to be wasters prior to the campaign, while those aged $65+$ are relatively less likely. The significant negative coefficient for females indicates that females are less likely to start as wasters than males. Hence, both age and gender influence the likelihood that an individual will be a food waster prior to the campaign. No significant effect for Private Garden was observed, which means respondents with access to a private garden are just as likely to start as wasters (or nonwasters) as those with no access.

The impact of covariates on transitions between states, relative to the probability of staying in the same state was examined (see Table 7). In the context of the current study this equates to testing which factors may increase or decrease the likelihood of wasters becoming non-wasters, or vice versa.

Table 7. Impact of covariates on transitions between states

\begin{tabular}{llll}
\hline Covariate & State (Pre) & $\begin{array}{l}\text { State (Post) } \\
\text { Non-Waster }\end{array}$ & Waster \\
\hline Age (18-44) & Non-Waster & & $-2.14(5.25)$ \\
& Waster & $-8.54(5.08)^{\wedge}$ & \\
Age (45-54) & Non-Waster & & $3.66(4.48)$ \\
& Waster & $-3.66(4.48)$ & \\
Age (55-64) & Non-Waster & & $-3.19(8.97)$ \\
Age $(65+)$ & Waster & $2.88(1.76)^{\wedge}$ & \\
& Non-Waster & &
\end{tabular}




\begin{tabular}{llll} 
Gender (Female) & Non-Waster & & $-2.74(2.76)$ \\
& Waster & $1.80(1.80)$ & \\
PrivateGarden (No) & Non-Waster & & $5.26(3.11)^{\wedge}$ \\
& Waster & $7.18(4.21)^{\wedge}$ & \\
Exposure & Non-Waster & & $-0.56(1.50)$ \\
& Waster & $-0.46(0.85)$ & $1.10(1.27)$ \\
Attitudes & Non-Waster & & \\
& Waster & $2.31(1.35)^{\wedge}$ & $-1.27(1.09)$ \\
SelfEfficacy & Non-Waster & & \\
& Waster & $7.18(4.07)^{\wedge}$ & $0.18(1.75)$ \\
SocialNorms & Non-Waster & & \\
\hline Displayed as Coefficient & (Standard Error), ${ }^{\wedge} \mathrm{p} \leq 0.1,{ }^{*} \mathrm{p} \leq 0.05,{ }^{* *} \mathrm{p} \leq 0.01,{ }^{* * *} \mathrm{p} \leq 0.001$
\end{tabular}

Results were not significant at the 0.05 probability level, which may be a result of the relatively low sample size. Many results had p-values around 0.06 or 0.07 , which given the sample size was considered to near traditional significance levels. Hence, results discussed are significant at the 0.1 level. The first notable results are that transitions from waster to non-waster are more likely among 55-64 year olds (based on the significant positive coefficient), but less likely among 18-44 year olds. That is, older individuals are more likely to improve their FV wastage compared to younger individuals.

Individuals with private gardens were likely to transition between states, from both non-wasters to wasters and vice versa. Significant results were observed for two of the three psychographic variables. Specifically, transitioning from waster to non-waster is positively associated with both attitudes and self-efficacy. In contrast, social norms had no impact on the likelihood of behavioural change. A summary of the Hidden Markov Model results is presented in Figure 1. This figure serves to graphically summarise the results presented in the tables above. The ovals represent the two latent states, including their respective sizes as reflected in Table 4. The curved arrows represent possible transitions between the states, including the possibility of remaining in the same state. The percentages attached to these arrows represent the transition probabilities, or rates of change, associated with each possible transition as reflected in Table 5. Finally, significant covariates (at the 0.1 level) highlighted in Table 7 are displayed next to 
each transition, with an upwards arrow demonstrating a positive impact, and a downwards arrow demonstrating a negative impact.

\section{Figure 1. Summary of Hidden Markov Model results}

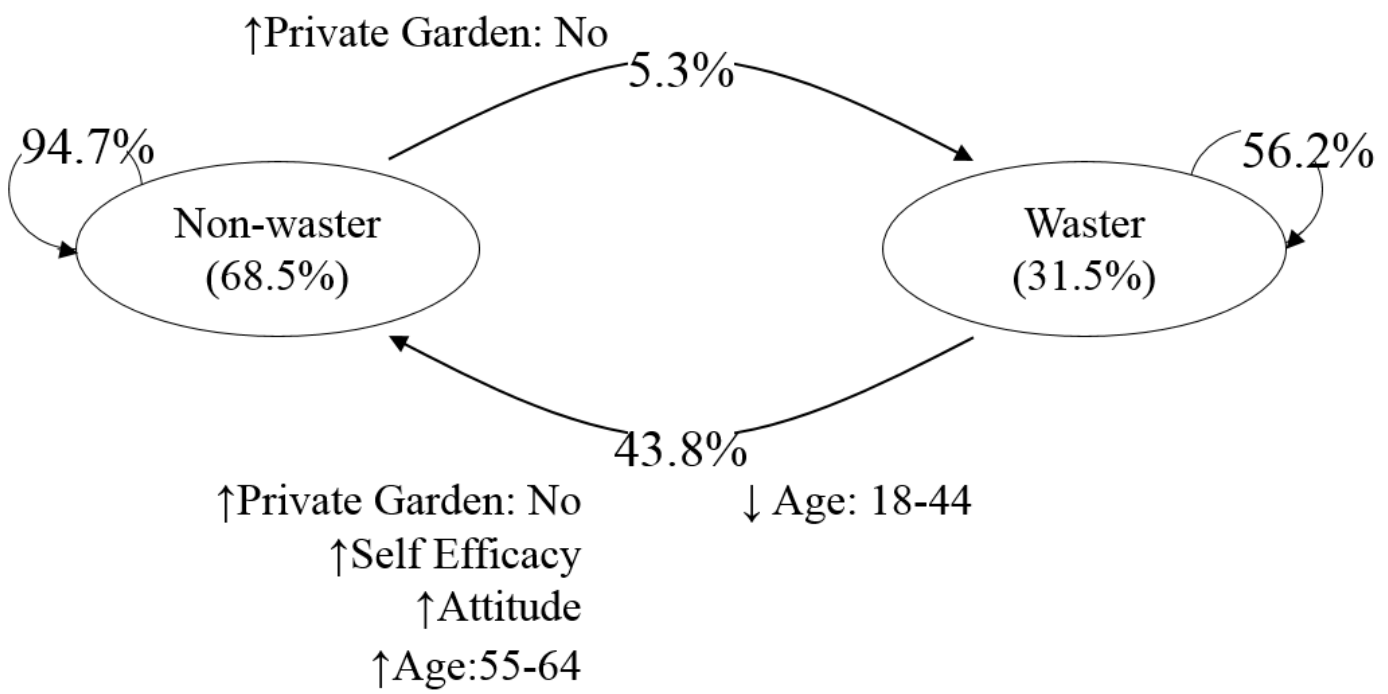

\section{Discussion}

This paper contributes to the literature in two ways. First, this paper applied Hidden Markov Modelling to explore the utility of this method, as an example of a dynamic model, to examine the causal determinants of positive and negative behavioural change. Application of Hidden Markov Modelling permitted an understanding of transition between states to be observed. A second contribution of this paper arises in the analytical insights gained. This study contributes to our understanding of food waste reduction behaviour identifying causal determinants of positive behavioural change (an increase in food waste reduction behaviour). Each contribution is discussed in turn in light of the literature.

\section{Behavioural states}

The dominant theoretical focus (see Brennan et al., 2014, Truong and Dang, 2017) centres research attention on identifying factors associated with behaviours typically drawing from cross-sectional data (for example, Huchting et al., 2008, Armitage, 2005). This research approach assists us to understand which conditions may need to exist for a behaviour to occur but they do not identify causal determinants of change. In response to calls to expand the research focus (see Michie and Prestwich, 2010) this paper drew on available data and applied HMM to examine changes in food waste behaviour following a two week pilot program 
directing analytical attention towards understanding the causal determinants of both behaviour and behavioural change. First, HMM identified two behavioural states and termed these FV wasters and non-FV wasters. A higher likelihood that non-wasters will remain non-wasters (after the program) was noted suggesting many individuals did not change, nor did they need to change given low FV waste behaviours reported by the majority in the sample prior to pilot program implementation. Next, the factors influencing the food waste behavioural state were examined. Analysis indicated a major causal factor for FV food wasting was age. Specifically, results indicated that individuals aged 45-54 were more likely to be food wasters than other age groups while females and individuals aged 65+ were less likely to be wasters. Theoretically derived psychographic measures (attitudes, self-efficacy and social norms) for the commonly used Theory of Planned Behaviour (Ajzen, 1991) did not explain FV waste group membership. Directing analytical focus to the behavioural states provides information that can guide program planning directing resources at those individual profiles most likely to be wasting food and results indicate that behaviour causal factors in this context were not psychographic determinants as suggested by Theory of Planned Behaviour.

\section{Transition and rates of behavioural change}

As stated previously, directing analytical focus at understanding the causal factors of behavioural change is recommended (see Michie and Prestwich, 2010 for a detailed explanation). In line with this recommendation HMM was applied to understand how, and why, people transition from one state to another (e.g. wasting food to not wasting food or vice versa). The transition probabilities estimated by the model identify the rates of change that occurred throughout the promotional period. A notably high probability of positive behavioural change was observed. However, it was also noted that the probability of individuals remaining in the same state (performing the same behaviour) is higher in both states than the probability of transitioning (behavioural change). This highlights a consistent challenge in social marketing of encouraging behavioural change within individuals. Importantly, through the HMM our study is able to examine causal determinants of behavioural change relative to stagnant behaviour. In the present study attitudes and self-efficacy were indicated as potential causal factors of FV waste change. Attitudes and perceived behavioural control explained why individuals transitioned from food wasting to non-food wasting, which was in line with the core program objective of leftover re-use (self-efficacy) through attractive and tasty recipes provided to the program group (attitudes). Importantly, these factors were significant for behavioural change, but not static behaviour as stated above. Hence, applying a dynamic model 
provides deeper insights into the role different variables have in behaviour compared to behaviour change.

Adoption of dynamic analytical approaches such as HMM extend understanding beyond dominant forms of outcome evaluation which deliver aggregate information. The strength of dynamic models such as HMM is identification of behavioural states as well as data on transitions between states, assisting program evaluators to understand the extent of behavioural change that can be expected and the factors causing behavioural change. This is especially relevant to the social marketing discipline, which has a dominant focus on cross-sectional designs, and even when a longitudinal design is employed, the most commonly used methodologies are static (such as ANOVA, for example). This paper makes an important contribution to the field applying a novel approach to assess behaviour change. An enhanced understanding of the causal determinants of change provides important data to inform dollar spend. In the current study investment focus on the factors causing non desired change could be avoided with funds directed towards factors causing desired behavioural change (FV wasters transitioning to non FV wasters). Identifying groups based on the behaviour of interest meets the segmentation principle recommended for social marketing, delivering a more nuanced means to direct strategy and investment, and theoretically an increased chance of achieving behavioural change (Carins and Rundle-Thiele, 2014, Xia et al., 2016).

\section{Implications for policy and practice}

The results of the current study indicate that FV food waste reduction can be changed through a focus on attitudes and beliefs about their own abilities. Therefore, based on this results of this study, social marketers seeking to reduce FV food waste should focus programs on selfefficacy and attitudes, although care should be taken prior to generalisation of these results and more tests within the same context are needed. In this pilot program recipe cards were

developed by leading regional chefs to provide recipes focussed on using contents that would already be in the household fridge. Daily cooking demonstrations were delivered during the 2week pilot program demonstrating how easy the recipes were to make and food samples were provided allowing individuals to trial the recipe. This pilot program provides one example of assisting to promote self-efficacy - one's confidence to re-use the items already available in the fridge. Additional efforts that could be considered in future food waste programs to further enhance self-efficacy include offering a cook book or cooking classes for purchase to ensure 
the program offers an essential exchange mechanism to avoid stigmatising or victimisation (Brennan et al., 2016).

The results of this study indicate potential utility of application of a dynamic approach to direct research and in turn practitioner focus to behaviour change and its determinants. The results of the current study suggest that social marketing definitions need to be revised from a focus on behaviour to an emphasis on behavioural change. By redirecting focus from behaviour to behavioural change, definitions and core focus will be altered ensuring that longitudinal designs are employed on all occasions to evaluate behavioural change. Dynamic modelling approaches such as HMM need to become mainstream practice in line with recommendations by Michie and Prestwich (2010) and Kriznik et al. (2018). Extending the evidence base to understand the causal determinants of change will enhance practice overall and ensure that monies invested in behavioural change programs are appropriately invested.

Understanding how to theorise, predict, measure and evaluate behaviour change is a hugely relevant issue. Various social marketing campaigns have been highly successful in the past but there is still limited understanding about why some campaigns work and why, despite repeated efforts other campaigns do not. By understanding the causal determinants of change, more effective behavioural change initiatives can be implemented across a broad spectrum of public health and environmental issues, from overweight and obesity, to mental health, problem gambling, greater equality for marginalised and vulnerable groups, sustainable use of resources and so on. Further, while the personal and social benefits of these changes are obvious effective delivery of change can deliver significant economic benefit.

\section{Limitations and future research directions}

The results of this study must be viewed in light of limitations. The sample size in this study was small particularly when considering that more than two-thirds of the sample were nonwasters prior to program implementation with low likelihoods of becoming food wasters, which it would be desired. As a result, a significance level of 0.1 was adopted in the present study based on the small sample size to illustrate the potential application of HMM to evaluate behavioural change. Therefore, the results must be treated with caution until larger sample sizes can be obtained. Future research drawing from larger sample sizes is recommended to draw definitive conclusions of HMM's potential to evaluate behavioural change. This study took a first step at looking at behaviour change from a dynamic perspective, however, it is recommended for future studies to also examine probabilities of long-term behaviour change 
and maintenance of behaviour. Expanding on this, future research is recommended to test the relevance and usefulness of alternative models of behaviour change, such as Latent Growth Curve Modelling.

In the current study, causal determinants of behaviour (initial food waste) were different from the causal determinants of behavioural change (transitions). Recall that psychographic constructs such as attitudes and self-efficacy did not initially explain FV food waste behaviour. Initial FV food waste behaviour was influenced by age and gender. In contrast to behaviour, causal determinants of behavioural change were increases in attitudes and self-efficacy suggesting the role these variables have to play in FV food waste reduction. This has important theoretical implications. Future research involving replication across contexts is recommended to develop a Theory of Behavioural Change. Additionally, the individual effects of each causal determinant were considered in this study. Future research may seek to examine whether there are important interactions between these factors.

This study was undertaken in the context of food waste and was focussed on changing individual behaviour. Extensions beyond the FV waste context are recommended for future research to examine the utility of HMM across a range of behavioural range contexts. Additionally, future research is recommended to include social and environmental behavioural change determinants into analysis to extend our understanding beyond individuals as recommended by Kriznik et al. (2018) and many other researchers.

The present study drew on available data to explore the utility of HMM for evaluating behaviour and behavioural change. Available data involved a repeated measure design and selfreport data. Self-report data for contexts such as food waste can be biased, given known biases such as socially desirable responding (Devaux and Sassi, 2015). Future research should obtain objective behavioural data in order to draw more definitive conclusions. A further limitation may arise from the combined use of the control and program use sample within a single HMM given that the control group is not expected to transition in the absence of an intervention. Additional research is recommended to understand the utility of including a control sample, or not, in transition modelling.

A related limitation based on available data is in the use of a single categorical variable to measure the behaviour of interest; food waste. Hidden Markov Models can include multiple indicator variables from various scale types (Vermunt, 2004). Further research should examine how results vary if multiple variables are used as indicators of the behaviour of interest. 
A final limitation of this study was that known biases such as socially desirable responding were not measured and this is recommended for future studies. The results of the current study indicated that almost half of individuals who were not exposed to the campaign still improved their food waste behaviour, which may be explained through a combination of the Hawthorne effect and social desirability bias (Chung and Monroe, 2003). Involvement in the study may have encouraged some participants to improve their FV food waste behaviour, while other respondents may have reported food waste reductions due to social desirability. Future research can determine the presence and absence of socially desirable responding and the Hawthorne effect through measurement of SDR and experimental designs.

This paper has demonstrated the role that HMM can play in delivering an assessment of a social marketing campaign to identify rates of positive (and negative) change and importantly to understand the factors predicting changes observed. Extending analytical focus to behavioural change ensures that resources can be directed to the causal determinants of behavioural change.

\section{Acknowledgements}

The data on which this article was based was funded by Redland City Council. The funders played no role in study design, collection, analysis, interpretation of data or in the decision to submit the paper for publication. They accept no responsibility for contents. 


\section{References}

Ajzen, I. (1991). "The theory of planned behavior". Organizational Behavior and Human Decision Processes, 50, 179-211.

Ajzen, I. (2015). "The theory of planned behaviour is alive and well, and not ready to retire: A commentary on sniehotta, presseau, and araújo-soares". Health Psychology Review, 9, 131-137.

Almestahiri, R., Rundle-Thiele, S., Parkinson, J. and Arli, D. (2017). "The use of the major components of social marketing: A systematic review of tobacco cessation programs". Social Marketing Quarterly, 23, 232-248.

Andreasen, A. R. (2002). "Marketing social marketing in the social change marketplace". Journal of Public Policy \& Marketing, 21, 3-13.

Armitage, C. J. (2005). "Can the theory of planned behavior predict the maintenance of physical activity?". Health Psychology, 24, 235.

Bandura, A. (1986). Social foundations of thought and action: A social cognitive theory, Prentice-Hall, Inc.

Brennan, L., Binney, W., Parker, L., Aleti, T. and Nguyen, D. (2014). Social marketing and behaviour change: Models, theory and applications, Edward Elgar Publishing.

Brennan, L., Previte, J. and Fry, M.-L. (2016). "Social marketing's consumer myopia: Applying a behavioural ecological model to address wicked problems". Journal of Social Marketing, 6, 219-239.

Carins, J. and Rundle-Thiele, S. (2014). "Eating for the better: A social marketing review (2000-2012)". Public Health Nutrition, 17, 1628-1639.

Chung, H., Park, Y. and Lanza, S. T. (2005). "Latent transition analysis with covariates: Pubertal timing and substance use behaviours in adolescent females". Statistics in Medicine, 24, 2895-2910.

Chung, J. and Monroe, G. S. (2003). "Exploring social desirability bias". Journal of Business Ethics, 44, 291-302.

Collins, L. M. and Lanza, S. T. (2010). Latent class and latent transition analysis: With applications in the social, behavioral, and health sciences, Hoboken, N.J., Wiley.

Davis, R., Campbell, R., Hildon, Z., Hobbs, L. and Michie, S. (2015). "Theories of behaviour and behaviour change across the social and behavioural sciences: A scoping review". Health Psychology Review, 9, 323-344.

Devaux, M. and Sassi, F. (2015). "Social disparities in hazardous alcohol use: Self-report bias may lead to incorrect estimates". The European Journal of Public Health, 26, 129134.

Fishbein, M. and Ajzen, I. (2011). Predicting and changing behavior: The reasoned action approach, Taylor \& Francis.

Gardner, B., de Bruijn, G. J. and Lally, P. (2012). "Habit, identity, and repetitive action: A prospective study of binge-drinking in uk students". British Journal of Health Psychology, 17, 565-581.

Huchting, K., Lac, A. and LaBrie, J. W. (2008). "An application of the theory of planned behavior to sorority alcohol consumption". Addictive Behaviors, 33, 538-551.

Jebb, A. T., Tay, L., Wang, W. and Huang, Q. (2015). "Time series analysis for psychological research: Examining and forecasting change". Frontiers in Psychology, $6,727$.

Kher, H. V. and Serva, M. A. (2014). "Changing the way we study change: Advocating longitudinal research in mis". ACM SIGMIS Database, 45, 45-60. 
Kriznik, N. M., Kinmonth, A. L., Ling, T. and Kelly, M. P. (2018). "Moving beyond individual choice in policies to reduce health inequalities: The integration of dynamic with individual explanations". Journal of Public Health.

Langeheine, R. and Van de Pol, F. (2002). "Latent markov chains". Applied Latent Class Analysis, 304-341.

Leatherdale, S. T., Brown, K. S., Carson, V., Childs, R. A., Dubin, J. A., Elliott, S. J., Faulkner, G., Hammond, D., Manske, S. and Sabiston, C. M. (2014). "The compass study: A longitudinal hierarchical research platform for evaluating natural experiments related to changes in school-level programs, policies and built environment resources". BMC Public Health, 14, 331.

Masyn, K. (2013). "Latent class analysis and finite mixture modeling". In: Little, T. D. (ed.) The oxford handbook of quantitative methods in psychology. Oxford University Press.

McCutcheon, A. L. (2002). "Basic concepts and procedures in single-and multiple-group latent class analysis". In: Hagenaars, J. A. and McCutcheon, A. L. (eds.) Applied latent class analysis. Cambridge University Press.

Michie, S., Johnston, M., Francis, J., Hardeman, W. and Eccles, M. (2008). "From theory to intervention: Mapping theoretically derived behavioural determinants to behaviour change techniques". Applied Psychology, 57, 660-680.

Michie, S. and Prestwich, A. (2010). "Are interventions theory-based? Development of a theory coding scheme". Health psychology, 29, 1.

Mihrshahi, S., Dobson, A. and Mishra, G. (2015). "Fruit and vegetable consumption and prevalence and incidence of depressive symptoms in mid-age women: Results from the australian longitudinal study on women's health". European Journal of Clinical Nutrition, 69, 585.

Netzer, O., Lattin, J. M. and Srinivasan, V. (2008). "A hidden markov model of customer relationship dynamics". Marketing Science, 27, 185-204.

Perugini, M. and Conner, M. (2000). "Predicting and understanding behavioral volitions: The interplay between goals and behaviors". European Journal of Social Psychology, 30, 705-731.

Ployhart, R. E. and Vandenberg, R. J. (2010). "Longitudinal research: The theory, design, and analysis of change". Journal of Management, 36, 94-120.

Prochaska, J. O., Velicer, W. F., Rossi, J. S., Goldstein, M. G., Marcus, B. H., Rakowski, W., Fiore, C., Harlow, L. L., Redding, C. A. and Rosenbloom, D. (1994). "Stages of change and decisional balance for 12 problem behaviors". Health Psychology, 13, 39.

Rhodes, R. E. and Courneya, K. S. (2003). "Investigating multiple components of attitude, subjective norm, and perceived control: An examination of the theory of planned behaviour in the exercise domain". British Journal of Social Psychology, 42, 129-146.

Rosenstock, I. M. (1974). "The health belief model and preventive health behavior". Health Education \& Behavior, 2, 354-386.

Rundle-Thiele, S., Schuster, L., Dietrich, T., Russell-Bennett, R., Drennan, J., Leo, C. and Connor, J. P. (2015). "Maintaining or changing a drinking behavior? Goka's shortterm outcomes". Journal of Business Research, 68, 2155-2163.

Schuster, L., Kubacki, K. and Rundle-Thiele, S. (2016). "Community-based social marketing: Effects on social norms". Journal of Social Marketing, 6, 193-210.

Shefferly, A., Scharf, R. J. and DeBoer, M. D. (2016). "Longitudinal evaluation of $100 \%$ fruit juice consumption on bmi status in 2-5-year-old children". Pediatric Obesity, 11, 221-227.

Singh, P. V., Tan, Y. and Youn, N. (2011). "A hidden markov model of developer learning dynamics in open source software projects". Information Systems Research, 22, 790807. 
Stancu, V., Haugaard, P. and Lähteenmäki, L. (2016). "Determinants of consumer food waste behaviour: Two routes to food waste". Appetite, 96, 7-17.

Truong, V. and Dang, N. (2017). "Reviewing research evidence for social marketing: Systematic literature reviews". In: Kubacki, K. and Rundle-Thiele, S. (eds.) Formative research in social marketing: Innovative methods to gain consumer insights. Singapore: Springer.

Vermunt and Magidson, J. (2013). "Technical guide for latent gold 5.0: Basic, advanced, and syntax". Belmont, MA: Statistical Innovations Inc.

Vermunt, J. K. (2004). "Latent markov model". In: Lewisbeck, M. S., Bryman, A. E. and Liao, T. F. (eds.) The sage encyclopedia of social sciences research methods. London: SAGE Publications.

Visser, I. (2011). "Seven things to remember about hidden markov models: A tutorial on markovian models for time series". Journal of Mathematical Psychology, 55, 403415.

Wang, M., Beal, D. J., Chan, D., Newman, D. A., Vancouver, J. B. and Vandenberg, R. J. (2017). "Longitudinal research: A panel discussion on conceptual issues, research design, and statistical techniques". Work, Aging and Retirement, 3, 1-24.

Waste and Resources Action Programme (2007). "We don't waste food: A householder survey".

Waste and Resources Action Programme (2010). "Monitoring awareness, claimed behaviour and satisfaction".

Xia, Y., Deshpande, S. and Bonates, T. (2016). "Effectiveness of social marketing interventions to promote physical activity among adults: A systematic review". Journal of Physical Activity and Health, 13, 1263-1274. 